\title{
Erratum to: Spectral caustic rendering of a homogeneous caustic object based on wavelength clustering and eye sensitivity
}

\author{
Budianto Tandianus • Henry Johan • \\ Hock Soon Seah • Feng Lin
}

Published online: 25 November 2014

(C) Springer-Verlag Berlin Heidelberg 2014

\section{Erratum to: Vis Comput \\ DOI 10.1007/s00371-014-1037-z}

The published paper contains a minor error (note the section highlighted with bold formatting) in the Abstract which we wish to make a correction on:

... Secondly, by considering the surrounding objects (their material reflectance from and visible surface area of the caustic objects) and light power, we compute the refinement amount of each wavelength cluster. Our accelerated ...
Should be written as:

... Secondly, by considering the surrounding objects (their material reflectance and their visible surface area from the caustic objects) and light power, we compute the refinement amount of each wavelength cluster. Our accelerated ...

The online version of the original article can be found under doi:10.1007/s00371-014-1037-z.

B. Tandianus $(\varangle)$

gameLAB, School of Computer Engineering Organization (SCE), Nanyang Technological University (NTU), Block NS4,

N4-B1b-13, 36 Nanyang Avenue, Singapore 639798, Singapore

e-mail: btandianus@ntu.edu.sg; budi0010@ntu.edu.sg

H. Johan

Fraunhofer IDM@NTU, Block NS1, N1-05-01, Nanyang Avenue,

Singapore 639798, Singapore

e-mail: henryjohan@ntu.edu.sg

H. S. Seah · F. Lin

School of Computer Engineering Organization (SCE),

Nanyang Technological University (NTU), Block NS4,

N4-02a-32, Nanyang Avenue, Singapore 639798, Singapore

H. S. Seah

e-mail: ashsseah@ntu.edu.sg

F. Lin

e-mail: asflin@ntu.edu.sg 